\title{
ON THE GENERIC NONEXISTENCE OF FIRST INTEGRALS
}

\author{
MIKE HURLEY
}

\begin{abstract}
The property of having no $C^{n}$ first integrals other than constants is shown to be generic in $\operatorname{Diff}^{r}(M)$ for each $r=1,2, \ldots$, where $n$ is the dimension of $M$.
\end{abstract}

Let $M$ be a compact Riemannian manifold, and consider the space $\operatorname{Diff}^{r}(M)$ of the $C^{r}$ diffeomorphisms from $M$ to itself with the $C^{r}$ topology. Peixoto has shown in [2] that the property of having no smooth first integrals other than constants is $C^{1}$ generic. This note describes how a combination of ideas of Arraut [1] and Takens [5] yields a proof that the nonexistence of (sufficiently) smooth, nonconstant first integrals is $C^{r}$ generic for any $r=1,2, \ldots, \infty$.

If $f: M \rightarrow M$ is a diffeomorphism, then a map $g: M \rightarrow R$ is a first integral of $f$ if (i) $g$ is constant along f-orbits: $g(f(x))=g(x)$ for all $x$ in $M$, and (ii) $g$ is not a constant. Peixoto's proof in [2] that the nonexistence of sufficiently smooth first integrals is a $C^{1}$ generic property is based on three observations. First, Sard's theorem implies that if $g$ is sufficiently smooth $\left(C^{n}\right.$ where $n$ is the dimension of $\left.M\right)$, and has no regular values in its image, then $g$ is constant. Second, by Pugh's general density theorem, the property of having the nonwandering set equal to the closure of the hyperbolic periodic points is generic in $\operatorname{Diff}^{1}(M)$ [3]. This is why Peixoto's argument works only to establish $C^{1}$ genericity. Third, if $g$ is a $C^{1}$ first integral of $f$ and $p$ is a hyperbolic periodic point of $f$, then $p$ is necessarily a critical point of $g$. Peixoto establishes this using a geometric argument based on the stable manifold theorem; a more elementary proof can be found in [4]. For $f$ satisfying Pugh's genericity condition, it follows from continuity that every nonwandering point of $f$ is a critical point of $g$. Since $M$ is compact, every level set of $g$ contains nonwandering points, so $g$ has no regular values in its image, and thus is constant.

By combining a theorem of Takens and an argument due to Arraut we can avoid using Pugh's theorem and so obtain the following result:

THEOREM. Suppose $M$ is a compact manifold of dimension $n$. For each $r=$ $1,2, \ldots, \infty$, there is a residual subset $S_{r}$ of $\operatorname{Diff}^{r}(M)$ with the property that if $f$ is in $S_{r}$ then no $C^{r}$ first integral of $f$ has any regular values in its image. In particular, if $r \geqslant n$, then no $f$ in $S_{r}$ has any nonconstant $C^{r}$ first integrals.

Received by the editors September 5, 1985.

1980 Mathematics Subject Classification (1985 Revision). Primary 58F35.

Key words and phrases. First integral, generic property.

(C)1986 American Mathematical Society $0002-9939 / 86 \$ 1.00+\$ .25$ per page 
Proof. Takens shows in [5] that there is a residual subset $S_{r}$ of $\operatorname{Diff}^{r}(M)$ with the property that if $f_{0}$ is in $S_{r}, \delta>0$, and $X$ is a nonempty, closed, $f_{0}$-invariant subset of $M$, then for any diffeomorphism $f$ sufficiently $C^{r}$ close to $f_{0}$, there is a nonempty, closed, $f$-invariant set $Y$ that is within $\delta$ of $X$ in the Hausdorff metric. In particular, if $U$ is open in $M$ and contains $X$, then any diffeomorphism $C^{r}$ close enough to $f_{0}$ has a nonempty, closed, invariant set contained in $U$.

Next we adapt an argument from [1] to show that no map in $S_{r}$ has a smooth first integral.

Lemma. Suppose $g: M \rightarrow R$ is $C^{r}(r \geqslant 1)$ and that $c=g(x)$ is a regular value of $g$. Let $N=g^{-1}(c)$. There is an open interval I containing $c$, a neighborhood $U$ of $N$ in $M$, and a $C^{r}$ diffeomorphism $h$ from $U$ onto $N \times I$ satisfying $h(x)=\left(h_{1}(x), g(x)\right)$, and $U=g^{-1}(I)$.

Proof of Lemma. Denote the Riemannian metric on $M$ by $\langle-,-\rangle$ and let $|-|$ be the associated norm. Take an open interval $I_{0}$, containing $c$, whose closure consists entirely of regular values of $g$. Define $U_{0}=g^{-1}\left(I_{0}\right)$. Consider the $C^{r-1}$ vector field $X=\operatorname{grad}(g) / \operatorname{grad}(g) \mid$ on $U_{0}$. By perturbing $X$ slightly in the $C^{r-1}$ topology we can find a $C^{\infty}$ vector field $Y$ defined on $U_{0}$ and satisfying

$$
\langle X(x), Y(x)\rangle>\frac{1}{2} \text { for all } x \text { in } U_{0} .
$$

Let $\psi$ denote the flow on $U_{0}$ induced by $Y$. By (1), $g$ is strictly increasing along forward $\psi$-orbits, and for each $q$ in $I_{0}$, the level set $g^{-1}(q)$ intersects each $\psi$-orbit in exactly one point. Consequently, for each $x$ in $U_{0}$ there is a uniquely defined real number $T(x)$ satisfying $g(\psi(T(x), x))=c$. By (1), the implicit function theorem applies to show that $T$ is $C^{r}$. Define $h: U_{0} \rightarrow N \times I_{0}$ by $h(x)=(\psi(T(x), x), g(x))$. $h$ is clearly $C^{r}$ and it is easy to check that $D h$ has full rank at each point of $N$, so by the inverse function theorem and the compactness of $N$, there is an open interval $I$ in $I_{0}$ such that $h$ is a $C^{r}$ diffeomorphism from $U$ onto $N \times I$, where $U=g^{-1}(I)$.

Now we return to the proof of the theorem. Suppose $f$ is in $\operatorname{Diff}^{r}(M)$ and that $g$ is constant along orbits of $f$. Assume that $g$ is also $C^{r}$, that $c$ is in the image of $g$ and is a regular value of $g$, so that $N=g^{-1}(c)$ is an embedded codimension one submanifold of $M$. Let $U, I$ and $h$ be as in the lemma, and select a $C^{\infty}$ map $j$ : $R \rightarrow R$ satisfying

(2) $j(t)>0$ for all $t$ in $I$, and $j(t)=0$ otherwise.

(3) id $+j$ is a diffeomorphism.

For $1>v>0$ define $f_{v}$ by

(4) $f_{v}=h^{-1} \circ(\mathrm{id}, \mathrm{id}+v \cdot j) \circ h \circ f$ on $U$, and by $f_{v}=f$ otherwise.

As $v$ decreases to $0, f_{v}$ converges to $f$ in the $C^{r}$ topology, and $g$ is strictly increasing along all $f_{v}$ orbits $(v>0)$ in $U$. If $I=(a, b)$, then it is easy to check that if $x$ is in $U$ and $v>0$, then $\left(f_{v}\right)^{k}(x)$ tends to $g^{-1}(b)$ as $k$ goes to infinity, and to $g^{-1}(a)$ as $k$ tends to negative infinity. Consequently, $U$ contains no nonempty, closed, $f_{v}$-invariant sets for any $1>v>0$. Since $U$ is an open neighborhood of the nonempty, closed, $f$-invariant set $N$, it follows that $f$ is not contained in Takens' residual set $S_{r}$. 


\section{REFERENCES}

1. J. L. Arraut, Note on structural stability, Bull. Amer. Math. Soc. 72 (1966), 542-544.

2. M. M. Peixoto, Qualitative theory of differential equations and structural stability, Differential Equations and Dynamical Systems (J. Hale and J. LaSalle, eds.), Academic Press, New York, 1967, pp. 469-480.

3. C. Pugh, An improved closing lemma and a general density theorem, Amer. J. Math. 89 (1967), 1010-1021.

4. C. Robinson, Generic properties of conservative systems, Amer. J. Math. 92 (1970), 562-603.

5. F. Takens, On Zeeman's tolerance stability conjecture, Manifolds-Amsterdam 1970, Lecture Notes in Math., vol. 197, Springer-Verlag, Berlin and New York, 1971, pp. 209-219.

Department of Mathematics and Statistics, Case Western Reserve University, Cleveland, OHIO 44106 